
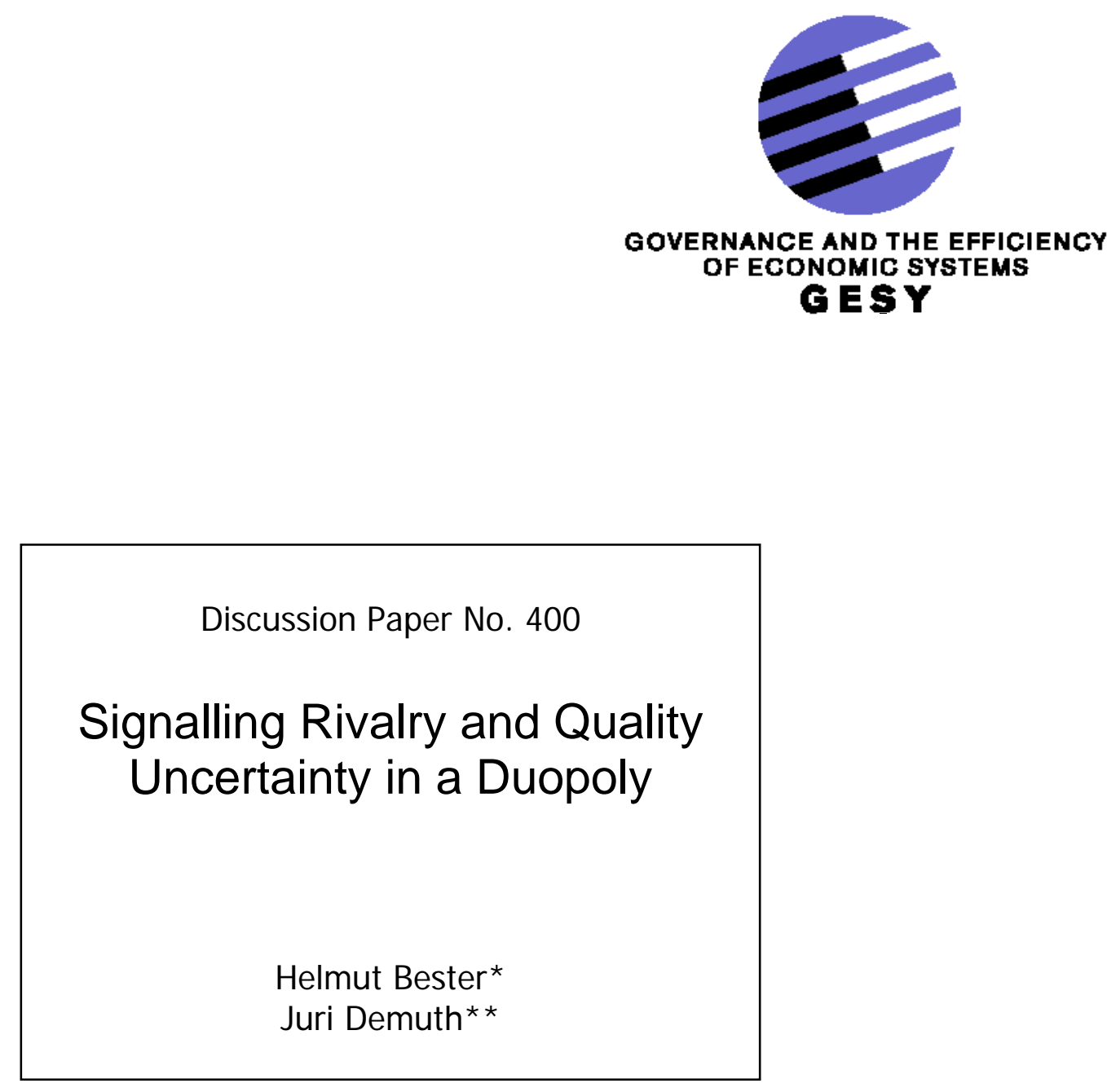

* Free University Berlin

** Free University Berlin

May 2013

Financial support from the Deutsche Forschungsgemeinschaft through SFB/TR 15 is gratefully acknowledged. 


\title{
SignALLING RivalRY AND QUALITY UnCERTAINTY IN A DUOPOLY*
}

\author{
Helmut Bester†and Juri Demuth
}

October 2011, revised May 2013

\begin{abstract}
This paper considers price competition in a duopoly with quality uncertainty. The established firm (the 'incumbent') offers a quality that is publicly known; the other firm (the 'entrant') offers a new good whose quality is not known by some consumers. The incumbent is fully informed about the entrant's quality. This leads to price signalling rivalry because the incumbent gains and the entrant loses if observed prices make the uninformed consumers more pessimistic about the entrant's quality. When the uninformed consumers' beliefs satisfy the 'intuitive criterion' and the 'unprejudiced belief refinement', prices signal the entrant's quality only in a two-sided separating equilibrium and are identical to the full information outcome.
\end{abstract}

Keywords: Quality uncertainty, Signalling, Oligopoly, Price competition

JEL Classification No.: D43, D82, L15

*Support by the German Science Foundation (DFG) through SFB/TR 15 is gratefully acknowledged.

${ }^{\dagger}$ Free University Berlin, Dept. of Economics, Boltzmannstr. 20, D-14195 Berlin (Germany); email: hbester@wiwiss.fu-berlin.de

${ }^{*}$ Free University Berlin, Dept. of Economics, Boltzmannstr. 20, D-14195 Berlin (Germany); email: juri.demuth@fu-berlin.de 


\section{Introduction}

It is well established that in markets with asymmetric information firms may use prices, possibly in conjunction with additional marketing devices, to signal quality information to uninformed market participants. In particular, if only some fraction of consumers is informed about quality, then firms may signal their qualities to the uninformed by setting prices higher than under perfect information. The idea is that high-quality firms suffer less from decreased sales to informed consumers due to price increases than low-quality firms. Therefore a highquality firm can separate itself by setting a high price which is not profitable to imitate for the low-quality firm. Signalling thus leads to distorted pricing and an inefficient reduction in the supply of high-quality goods.

This paper studies an extension of the standard price signalling model to a durable goods duopoly. In this environment the informative signalling equilibrium is free of distortions and identical to the perfect information equilibrium. We obtain this conclusion for a horizontally and vertically differentiated duopoly market with price-setting competitors engaging in a game of signalling rivalry: An established firm ("incumbent"), whose quality is known by all market participants, faces a competitor ("entrant") who is either supplying the same quality as the incumbent or a superior quality acquired through some product innovation. Both firms and some fraction of consumers know the entrant's quality. The uninformed consumers use prices set by both firms to infer quality information. An important feature of price competition is that the two firms have opposing interests in conveying information, because the incumbent gains and the entrant loses when observed prices make the uninformed consumers more pessimistic about the entrant's quality.

In our model consumers are confronted with two price signals concerning a single uncertain variable, the entrant's quality. For the analysis of equilibrium, we apply two standard refinements for the uninformed consumers' out-of-equilibrium beliefs. First, we use the 'intuitive criterion' of Cho and Kreps (1987). Second, in situations where one of the firms' pricing is informative we adopt the 'unprejudiced belief criterion' of Bagwell and Ramey (1991) to the pricing strategy of its competitor, because the intuitive criterion is no longer applicable. Under the unprejudiced belief criterion the consumers trust in the price signal of the nondeviating competitor whenever only one of the two firms selects an out-of-equilibrium price. This means that, given the other firm plays an equilibrium separating strategy, a deviating firm cannot influence beliefs by deviating to a non-equilibrium price and therefore always sets its best response price as under perfect information.

The unprejudiced belief criterion therefore excludes all separating equilibria with prices distorted from full-information prices. We show that these prices constitute the unique separating equilibrium outcome in our model as long as the fraction of informed consumers is not too small. If only rather few consumers are informed, there is no informative equilibrium. The reason is that either the low-type entrant could gain by deviating to the high-type equilibrium price or the incumbent playing against the high-type would deviate to the low-type equilibrium price. Thus the firms' price signals would become contradictory: The entrant 
would signal that his quality is high and the incumbent that the entrant's quality is low.

\section{Related Literature}

The standard prediction of the literature on price signalling is that quality uncertainty leads to distorted pricing for signalling purposes. The earliest contributions to this literature consider a market with a single seller. For example, Milgrom and Roberts (1986) show that a monopolist may use price and advertisement to convince consumers of the quality of a newly introduced product. In their model, which is based on repeat purchases of a non-durable good, prices can be distorted up- or downwards depending on expectations over future sales. Bagwell and Riordan (1991) consider a monopolist who produces a durable good whose quality may be high or low. The existence of informed consumers and cost differences between qualities allow the monopolist to signal high quality through an upward distorted price ${ }^{1}$ Basically, our model extends Bagwell and Riordan (1991) to a horizontally differentiated duopoly in which one of the two firms offers a quality that is known to the competitor but not to all consumers.

One strand of the literature extends the analysis of price signalling to oligopolistic markets under the assumption that firms have private information only about their own quality. They are not informed about the other firms and, therefore, have the same prior about their competitors' qualities as the uninformed consumers. Daughety and Reinganum (2007) and Daughety and Reinganum (2008) examine a horizontally and vertically differentiated duopoly and $n$-firm oligopoly, respectively. Price setting takes into account the ex-ante probabilities of rivals to be high- or low-quality types. Separating equilibria imply upward distorted prices, increasing in the ex-ante probability of firms being high-types. Similarly, Janssen and Roy (2010) show for a homogenous oligopoly that fully revealing mixed strategy equilibria exist in which high-types distort prices upward and low-types randomize prices over an interval, thereby generating sufficient rents to avoid mimicry of the high-types.

Closer related to the information structure in our model is the other strand of the literature that assumes the oligopolists to be informed about their rivals' qualities. Hertzendorf and Overgaard (2001a) analyze price setting and advertising in a duopoly. To keep the analysis tractable, they assume that qualities are perfectly negatively correlated and consumers only know that one firm offers high quality and the other low quality. They apply two refinements that lead to a unique separating and a unique pooling equilibrium. In the separating equilibrium, a high degree of vertical differentiation leads to upwards distorted prices and a low degree to downward distorted prices. Yehezkel (2008) introduces some informed consumers into a similar model and examines how pricing and advertising strategies depend on the fraction of informed consumers.

In Fluet and Garella (2002) the ex ante distribution of the firm's qualities is such that either both firms offer low quality or one firm offers low and the other high quality. The

\footnotetext{
${ }^{1}$ Linnemer (2002) shows that in the same setup it would be in some cases more profitable for the high-type firm to combine price and advertising signals.
} 
authors avoid the use of selection criteria and find multiple separating and pooling equilibria. For small quality differences separation can only be achieved with a combination of upward distorted prices and advertisement. This result is similar to a finding by Hertzendorf and Overgaard (2001b), who show that fully revealing separating equilibria satisfying the unprejudiced belief condition do not exist.

These papers differ from our model in that they consider product differentiation only in the vertical dimension. This implies that the duopolists have a common interest in signalling different qualities since they earn zero profits if consumers believe that they both offer the same expected quality. In our model of signalling rivalry such a common interest does not exist because consumer preferences are differentiated horizontally between the firms, and in the vertical dimension all consumers have identical preferences. As a consequence, the incumbent always prefers the consumers to believe that the entrant's quality is identical to his own quality, whereas the entrant gains by convincing the consumers that he offers a superior quality. Another feature that distinguishes our model from the above literature is that the duopolists are not in a symmetric position. Consumers are uninformed only about the entrant's and not about the incumbent's quality. They interpret the prices of both firms as signals only about the entrant's quality. In our analysis, we do not address expenditures on directly uninformative advertising as an additional signal. Since under our belief refinements only the full-information equilibrium without distortions survives, there is no role for dissipative advertising in equilibrium.

From a methodological perspective our analysis is closely related to Bagwell and Ramey (1991) and Schultz (1999). They study limit pricing by two incumbents to affect the entry decision of a third firm. The incumbents' prices signal their information about an industrywide parameter. The third firm enters the market only if it concludes that the probability of a favorable state is sufficiently high. In the paper by Bagwell and Ramey (1991) the competitors have a common interest, both want to signal an unfavorable state in order to prevent entry. Introducing the unprejudiced belief refinement, the authors find that only non-distorted separating equilibria exist. Further, under additional assumptions the intuitive criterion of Cho and Kreps (1987) eliminates all equilibria with pooling. By applying the same belief refinements to our context, we arrive at similar conclusions for the qualitative features of equilibrium. Schultz (1999) considers a variation of Bagwell and Ramey (1991) where the incumbents have conflicting interests, i.e. one incumbent prefers the entrant to stay out of the market, whereas its competitor profits from entry. Again, separating equilibrium prices are not distorted. But due to signalling rivalry these equilibria only exist if the effect of entry on the incumbent's profits is relatively small. We obtain a related result in our model when the fraction of informed consumers is rather small.

This paper is organized as follows. In Section 2 we describe the model and, as a reference point, we derive the equilibrium under full information. Section 3 defines the Perfect Bayesian Equilibrium and explains the belief refinements of our analysis. In Section 4 we show that under our refinements only the full information equilibrium prices can survive in an informative equilibrium and that such an equilibrium exists if the fraction of informed 
consumers is not too small. Section 5 provides concluding remarks. The proofs of all formal results are relegated to an appendix in Section 6 .

\section{The Model}

We employ the demand structure of the standard Hotelling (1929) duopoly with the modification that the two firms may offer different qualities. While one of the firms offers a quality that is publicly known by all market participants, the quality of the other firm is not known to some share of consumers. This environment may describe a market in which a new firm has entered to compete against an already established firm. In such a market it is reasonable to assume that consumers are informed about the established firm's quality through their prior experience, but that some consumers are uncertain about the new firm's product quality. In the terminology of Nelson (1970), the new good is an experience good so that an uninformed consumer learns its true quality only after purchase. For convenience, in what follows we call the established firm the incumbent and the new firm the entrant. Our formal analysis focuses on post-entry price competition, but in the conclusions in Section 5 we indicate some implications for market entry decisions.

The incumbent firm is fully informed about the true quality of the entrant. The uninformed consumers use the firms' prices to draw inferences about the entrant's quality. Accordingly, the price setting behavior of both firms takes into account that prices are quality signals.

There is a unit mass of consumers whose preference characteristic $x$ is uniformly distributed on the interval $[0,1]$. Each consumer purchases at most one unit of the good from either the incumbent $I$ or the entrant $E$. Given the incumbent's quality $q_{I}$ and the entrant's (expected) quality $q_{E}$, the valuation of a consumer with characteristic $x \in[0,1]$ is

$$
v_{I}(x)=q_{I}-t x, \quad v_{E}(x)=q_{E}-t(1-x)
$$

for the incumbent's and the entrant's good. The parameter $t$ reflects the degree of horizontal product differentiation. The two firms are also vertically differentiated if $q_{I} \neq q_{E}$. But the quality differential between the two firms affects the taste of all consumers in the same way, independently of their characteristic $x$. This aspect distinguishes our model from the price signalling models of Hertzendorf and Overgaard (2001a) and Fluet and Garella (2002) who similarly to Shaked and Sutton (1982) assume that consumers differ in their valuation of quality and that the goods are not horizontally differentiated. In what follows, we assume that the firms' qualities are sufficiently high so that each consumer buys one unit of the good.

All consumers observe the incumbent's price $p_{I}$ and the entrant's price $p_{E}$. The critical consumer type $\tilde{x}$, who is indifferent between purchasing from firm $I$ and firm $E$, is then determined by $v_{I}(\tilde{x})-p_{I}=v_{E}(\tilde{x})-p_{E}$, and by (1) we have

$$
\tilde{x}\left(p_{I}, p_{E}, q_{E}-q_{I}\right)=\max \left\{0, \min \left\{\frac{p_{E}-p_{I}-\left(q_{E}-q_{I}\right)+t}{2 t}, 1\right\}\right\} .
$$


All consumers with $x<\tilde{x}$ optimally buy the incumbent's good, whereas consumers with $x>\tilde{x}$ purchase from the entrant.

There are two possible qualities, $q_{L}$ and $q_{H}$, with $0<q_{L}<q_{H}$. The incumbent's quality is commonly known to be $q_{I}=q_{L}$. There is uncertainty, however, about the entrant's quality. Its quality is $q_{E}=q_{H}$ with ex ante probability $\lambda$ and $q_{E}=q_{L}$ with probability $1-\lambda$. One interpretation is that with probability $\lambda$ the entrant has realized a product innovation which increases the 'standard' quality $q_{L}$ by the amount $q_{H}-q_{L}$. We normalize the unit cost of producing quality $q_{L}$ to zero and assume that the unit cost of producing quality $q_{H}$ is $c>0$.

By (2) only the quality differential between the two firms affects the consumers' demand decisions. Therefore, we can simplify notation by defining

$$
\Delta \equiv q_{H}-q_{L}
$$

We assume that the high quality entrant has a competitive advantage because $c<\Delta$.

Both firms observe the realization of $q_{E}$ before setting prices. In addition some fraction $\gamma \in(0,1)$ of consumers becomes informed about the entrant's true quality before making demand decisions. Each consumer type $x$ is equally likely to be informed. This means that in each subset of the consumers' characteristic set $[0,1]$ the fraction of informed consumers is identically equal to $\gamma$.

The uninformed consumers use the observed prices $p_{I}$ and $p_{E}$ to draw inferences about the entrant's quality. We denote their posterior belief that the entrant's quality is $q_{E}=q_{H}$ by $\mu \in[0,1]$. Thus the uninformed consumers anticipate that the entrant offers the expected quality $\mu q_{H}+(1-\mu) q_{L}=q_{L}+\mu \Delta$. Since consumers are risk-neutral with respect to quality, for given prices $p_{I}$ and $p_{E}$ their demand decisions depend only on the expected quality difference between the two sellers.

In the uninformed consumers' expectation the quality difference $q_{E}-q_{I}$ is always equal to $\mu \Delta$, independently of the entrant's true quality. If the entrant's quality is $q_{E}=q_{L}$, the informed consumers know that $q_{E}-q_{I}=0$. Therefore, by (2) the incumbent's and the entrant's market shares, $D_{I L}$ and $D_{E L}$, are given by

$$
\begin{aligned}
D_{I L}\left(p_{I}, p_{E}, \mu\right) & =\gamma \tilde{x}\left(p_{I}, p_{E}, 0\right)+(1-\gamma) \tilde{x}\left(p_{I}, p_{E}, \mu \Delta\right), \\
D_{E L}\left(p_{I}, p_{E}, \mu\right) & =1-D_{I L}\left(p_{I}, p_{E}, \mu\right) .
\end{aligned}
$$

If $q_{E}=q_{H}$, the informed consumers know that $q_{E}-q_{I}=\Delta$. In this case, the two sellers' market shares, $D_{I H}$ and $D_{E H}$, are equal to

$$
\begin{aligned}
D_{I H}\left(p_{I}, p_{E}, \mu\right) & =\gamma \tilde{x}\left(p_{I}, p_{E}, \Delta\right)+(1-\gamma) \tilde{x}\left(p_{I}, p_{E}, \mu \Delta\right), \\
D_{E H}\left(p_{I}, p_{E}, \mu\right) & =1-D_{I H}\left(p_{I}, p_{E}, \mu\right) .
\end{aligned}
$$

Therefore, if the entrant's quality is $q_{E}=q_{L}$, the incumbent's profit is $\Pi_{I L}=p_{I} D_{I L}$ and the entrant's profit $\Pi_{E L}=p_{E} D_{E L}$. If $q_{E}=q_{H}$, the duopolists' profits are $\Pi_{I H}=p_{I} D_{I H}$ and $\Pi_{E H}=\left(p_{E}-c\right) D_{E H}$. 
Note that for all $\gamma \in(0,1)$ it is the case that

$$
\frac{\partial \Pi_{E L}}{\partial \mu} \geq 0, \quad \frac{\partial \Pi_{E H}}{\partial \mu} \geq 0 ; \quad \frac{\partial \Pi_{I L}}{\partial \mu} \leq 0, \quad \frac{\partial \Pi_{I H}}{\partial \mu} \leq 0 .
$$

Irrespective of the true quality, the entrant never loses and the incumbent never gains when the uninformed consumers raise their belief that the entrant offers high quality. Since these consumers interpret the firms' prices as quality signals, price competition entails a signalling rivalry: The entrant has an incentive to choose a price that indicates high quality. This is in conflict with the incumbent's interest to convince consumers that the entrant offers low quality.

Before analyzing how the duopolists' signalling rivalry affects their price competition, we briefly describe the equilibrium under full information. The firms compete by simultaneously setting prices and their pricing strategies are contingent on the entrant's quality. If $q_{E}=q_{L}$, we denote the incumbent's and the entrant's price by $p_{I L}$ and $p_{E L}$, respectively; if $q_{E}=q_{H}$ the firms' prices are denoted by $p_{I H}$ and $p_{E H}$. When all consumers know the entrant's quality, the firms' profits can be calculated by setting $\mu \equiv 0$ for $q_{E}=q_{L}$ and $\mu \equiv 1$ for $q_{E}=q_{H}{ }^{2}$ The full information equilibrium prices $\hat{p}=\left(\left(\hat{p}_{I L}, \hat{p}_{E L}\right),\left(\hat{p}_{I H}, \hat{p}_{E H}\right)\right)$ are then defined by the conditions for profit maximization so that for all $p \geq 0$

$$
\begin{aligned}
\Pi_{I L}\left(\hat{p}_{I L}, \hat{p}_{E L}, 0\right) & \geq \Pi_{I L}\left(p, \hat{p}_{E L}, 0\right), \quad \Pi_{E L}\left(\hat{p}_{I L}, \hat{p}_{E L}, 0\right) \geq \Pi_{E L}\left(\hat{p}_{I L}, p, 0\right), \\
\Pi_{I H}\left(\hat{p}_{I H}, \hat{p}_{E H}, 1\right) & \geq \Pi_{I H}\left(p, \hat{p}_{I H}, 1\right), \quad \Pi_{E H}\left(\hat{p}_{I H}, \hat{p}_{E H}, 1\right) \geq \Pi_{E H}\left(\hat{p}_{I H}, p, 1\right) .
\end{aligned}
$$

From the corresponding first-order conditions one can easily derive the full information reaction functions

$$
\begin{aligned}
R_{I L}\left(\hat{p}_{E L}\right) & =\max \left\{\frac{\hat{p}_{E L}+t}{2}, \hat{p}_{E L}-t, 0\right\} \\
R_{E L}\left(\hat{p}_{I L}\right) & =\max \left\{\frac{\hat{p}_{I L}+t}{2}, \hat{p}_{I L}-t, 0\right\} \\
R_{I H}\left(\hat{p}_{E H}\right) & =\max \left\{\frac{\hat{p}_{E H}+t-\Delta}{2}, \hat{p}_{E H}-t-\Delta, 0\right\} \\
R_{E H}\left(\hat{p}_{I H}\right) & =\max \left\{\frac{\hat{p}_{I H}+t+\Delta+c}{2}, \hat{p}_{I H}-t+\Delta, c\right\} .
\end{aligned}
$$

The solution of (8)-(11) depends on the high quality entrant's competitive advantage. If $\Delta-c<3 t$ the innovation is non-drastic and the incumbent gets positive profits even when the entrant offers high quality. Otherwise, if $\Delta-c \geq 3 t$, the innovation is drastic and implies that the incumbent's market share is zero. The full information equilibrium prices in these two cases are

$$
\begin{array}{cl}
\hat{p}_{I L}=t, \hat{p}_{E L}=t, \hat{p}_{I H}=t-(\Delta-c) / 3, \hat{p}_{E H}=t+(\Delta+2 c) / 3 & \text { if } \quad \Delta-c<3 t, \\
\hat{p}_{I L}=t, \hat{p}_{E L}=t, \hat{p}_{I H}=0, \hat{p}_{E H}=\Delta-t & \text { if } \quad \Delta-c \geq 3 t .
\end{array}
$$

\footnotetext{
${ }^{2}$ This is equivalent to setting $\gamma \equiv 1$.
} 
If $q_{E}=q_{L}$, both firms charge the same price and have the same market share $D_{I L}=D_{E L}=1 / 2$. If $q_{E}=q_{H}$, the incumbent is disadvantaged against the entrant and, even though he sets a lower price, his market share for non-drastic innovations $D_{I H}=(3 t-\Delta+c) /(6 t)$ is smaller than the entrant's share $D_{E H}=(3 t+\Delta-c) /(6 t)$. For drastic innovations we have $D_{I H}=0$ and $D_{E H}=1$.

\section{Equilibrium and Belief Restrictions}

We envisage the market to operate in three stages. First, both firms and a fraction $\gamma$ of consumers observe the realization of the entrant's quality. Second, the firms compete by simultaneously setting prices. Finally, in the third stage the uninformed consumers use observed prices to update their beliefs about the entrant's quality, and all consumers decide whether to buy from the incumbent or the entrant.

In what follows we study pricing strategies of the firms and consumer beliefs that constitute a Perfect Bayesian Equilibrium of this game. The firms choose their prices contingent on their information about the entrant's quality, and the uninformed consumers' posterior probability of facing the high quality entrant is a function of the firms' prices. In equilibrium, each firm's price maximizes its profit and the uninformed consumer's posterior belief is consistent with Bayesian updating. ${ }^{3}$

More formally, $\left(p^{*}, \mu^{*}(\cdot)\right)=\left(\left(p_{I L}^{*}, p_{E L}^{*}\right),\left(p_{I H}^{*}, p_{E H}^{*}\right), \mu^{*}(\cdot)\right)$ with $\mu^{*}: \mathbf{R}_{+}^{2} \rightarrow[0,1]$ is a Perfect Bayesian Equilibrium (PBE) if

(a) for $Q=L, H$

$$
\begin{aligned}
& p_{I Q}^{*}=\operatorname{argmax}_{p} \Pi_{I Q}\left(p, p_{E Q}^{*}, \mu^{*}\left(p, p_{E Q}^{*}\right)\right), \\
& p_{E Q}^{*}=\operatorname{argmax}_{p} \Pi_{E Q}\left(p_{I Q}^{*}, p, \mu^{*}\left(p_{I Q}^{*}, p\right)\right),
\end{aligned}
$$

and (b)

$$
\begin{array}{r}
\mu^{*}\left(p_{I L}^{*}, p_{E L}^{*}\right)=1-\mu^{*}\left(p_{I H}^{*}, p_{E H}^{*}\right)=0, \quad \text { if } \quad p_{I L}^{*} \neq p_{I H}^{*} \quad \text { or } \quad p_{E L}^{*} \neq p_{E H}^{*}, \\
\mu^{*}\left(p_{I L}^{*}, p_{E L}^{*}\right)=\mu^{*}\left(p_{I H}^{*}, p_{E H}^{*}\right)=\lambda, \quad \text { if } \quad p_{I L}^{*}=p_{I H}^{*} \text { and } p_{E L}^{*}=p_{E H}^{*} .
\end{array}
$$

Equilibrium conditions (13) and (14) state that, for each quality $q_{E} \in\left\{q_{L}, q_{H}\right\}$, the incumbent and the entrant choose their prices to maximize profits, taking the competitor's price and the uninformed consumers' beliefs $\mu^{*}(\cdot)$ as given. Equilibrium conditions (15) and (16) require that on the equilibrium path the buyers' belief is consistent with Bayes' rule. The buyers become fully informed about the entrant's true quality not only in a two-sided separating equilibrium, where $p_{i L}^{*} \neq p_{i H}^{*}$ for both $i \in\{I, E\}$, but also in a one-sided separating equilibrium, where $p_{i L}^{*} \neq p_{i H}^{*}$ for some $i \in\{I, E\}$ and $p_{j L}^{*}=p_{j H}^{*}$ for $j \neq i$. Prices remain uninformative only

\footnotetext{
${ }^{3}$ We restrict ourselves to pure strategy equilibria.
} 
if $p_{i L}^{*}=p_{i H}^{*}$ for both $i \in\{I, E\}$. In such a pooling equilibrium the posterior belief is equal to the a priori probability $\lambda$. Since we are interested in the existence and properties of informative equilibria, we ignore pooling in our subsequent analysis.

By (13) and (14), the uninformed consumers' quality expectations affect the duopolists' pricing decisions. But, conditions (15) and (16) impose restrictions on expectations only for prices that are actually chosen in equilibrium. Since out-of-equilibrium beliefs remain arbitrary, there are multiple equilibria, which are a typical feature of signalling games. This is so because the profit of a deviation from the equilibrium price depends on the uninformed consumers' interpretation of this deviation. For example, the incumbent may be deterred from changing its price simply because consumers would interpret this as a signal that the entrant's quality is high. Similarly, the entrant may be kept from changing its price if consumers view this as a signal of low quality. Without restrictions on consumer beliefs multiple equilibria with both upward and downward distorted prices can be found .

To avoid this problem, the literature usually applies refinements that impose restrictions on out-of-equilibrium beliefs. A prominent refinement is the 'intuitive criterion' of Cho and Kreps (1987), which has been used in a variety of price signalling games $4^{4}$ Unfortunately, this criterion is not generally applicable in the present context because it is defined for signalling games where each player has private information only about his own and not the other players' characteristics. In our model, however, the duopolists have common private information and not only the entrant's but also the incumbent's price may signal the entrant's quality. Therefore, the intuitive criterion cannot be used in our model if both firms' prices are informative. Nonetheless, it remains applicable if one of the firms' equilibrium prices are uninformative, i.e. if $p_{i L}^{*}=p_{i H}^{*}$ for some $i \in\{I, E\}$. In this case, the intuitive criterion can be used to refine beliefs for out-of-equilibrium prices of firm $j \neq i$.

Consider the incumbent in a situation where the entrant charges $p_{E L}^{*}=p_{E H}^{*}$ and the incumbent knows that the entrant's quality is low. Suppose the incumbent wishes to deviate to some price $p_{I}$ if the uninformed consumers interpret $p_{I}$ as a signal that indicates a low quality entrant. Then the idea of the intuitive criterion is that $p_{I}$ should indeed convince the consumers that the entrant offers low quality if the following is true: If the incumbent knew that the entrant's quality is high, he would not gain from deviating to $p_{I}$ even if the consumers would respond favorably for the incumbent by believing that $p_{I}$ indicates a low quality entrant.

An analogous argument applies to the high quality entrant in a situation where the incumbent's pricing $p_{I L}^{*}=p_{I H}^{*}$ reveals no information. In this case, the intuitive criterion requires the uninformed consumers to believe that a price $p_{E}$ signals high quality if for this belief deviating to $p_{E}$ is profitable only for the high quality entrant and not for the low quality entrant.

More formally, the PBE $\left(p^{*}, \mu^{*}(\cdot)\right)$ satisfies the intuitive criterion if the following two conditions (a) and (b) are satisfied:

\footnotetext{
${ }^{4}$ See, for example, Bagwell and Riordan (1991), Bagwell and Ramey (1991), Bester (1993), Bester and Ritzberger (2001).
} 
(a) If $p_{E L}^{*}=p_{E H}^{*}=p_{E}^{*}$, then $\mu^{*}\left(p_{I}, p_{E}^{*}\right)=0$ for all $p_{I}$ such that

$$
\Pi_{I H}\left(p_{I}, p_{E}^{*}, 0\right) \leq \Pi_{I H}\left(p_{I H}^{*}, p_{E}^{*}, \mu^{*}\left(p_{I H}^{*}, p_{E}^{*}\right)\right)
$$

and

$$
\Pi_{I L}\left(p_{I}, p_{E}^{*}, 0\right)>\Pi_{I L}\left(p_{I L}^{*}, p_{E}^{*}, \mu^{*}\left(p_{I L}^{*}, p_{E}^{*}\right)\right) .
$$

(b) If $p_{I L}^{*}=p_{I H}^{*}=p_{I}^{*}$, then $\mu^{*}\left(p_{I}^{*}, p_{E}\right)=1$ for all $p_{E}$ such that

$$
\Pi_{E L}\left(p_{I}^{*}, p_{E}, 1\right) \leq \Pi_{E L}\left(p_{I}^{*}, p_{E L}^{*}, \mu^{*}\left(p_{I}^{*}, p_{E L}^{*}\right)\right)
$$

and

$$
\Pi_{E H}\left(p_{I}^{*}, p_{E}, 1\right)>\Pi_{E H}\left(p_{I}^{*}, p_{E H}^{*}, \mu^{*}\left(p_{I}^{*}, p_{E H}^{*}\right)\right) .
$$

As a refinement for situations where firm $i \in\{I, E\}$ defects from the equilibrium and firm $j \neq i$ uses a separating strategy $p_{j L}^{*} \neq p_{j H}^{*}$, we employ the 'unprejudiced belief criterion' introduced by Bagwell and Ramey (1991). The basic idea of this criterion is that upon observing an out-of-equilibrium price pair $\left(p_{I}, p_{E}\right)$ the uninformed consumers rationalize their observation with the fewest number of deviations from the equilibrium strategies. Therefore, if a price pair occurs where one of the prices is out-of-equilibrium while the other price belongs to the separating pricing strategy of the competitor, the consumers believe that the entrant's quality is signaled by the competitor.

Actually, since there are only two types of the entrant, in our context it is sufficient to consider a simplified version of the unprejudiced belief criterion: If only the entrant chooses an out-of-equilibrium price $p_{E}$ and the incumbent's equilibrium price $p_{I H}^{*}$ indicates a high quality entrant, then the uninformed consumers should conclude that the entrant offers high quality; there are no belief restrictions if the incumbent's price $p_{I L}^{*}$ signals low quality. Indeed, a high quality signal of the incumbent looks rather convincing since it is against his interest to admit that his competitor offers a superior good. An analogous reasoning applies when the uninformed consumers conjecture that the price $p_{I}$ constitutes a unilateral deviation by the incumbent. In this situation, they should infer from the entrant's price $p_{E L}^{*}$ that his quality is low; there are no belief restrictions if the entrant's price is $p_{E H}^{*}$. Again, this seems plausible because expecting high quality makes little sense if the entrant acknowledges that his quality is low.

More formally, the PBE $\left(p^{*}, \mu^{*}(\cdot)\right)$ satisfies the (simplified) unprejudiced belief criterion if the following two conditions (a) and (b) are satisfied:

(a) If $p_{I L}^{*} \neq p_{I H}^{*}$, then $\mu^{*}\left(p_{I H}^{*}, p_{E}\right)=1$ for all $p_{E} \neq p_{E L}^{*}$.

(b) If $p_{E L}^{*} \neq p_{E H}^{*}$, then $\mu^{*}\left(p_{I}, p_{E L}^{*}\right)=0$ for all $p_{I} \neq p_{I H}^{*}$.

Notice that in a two-sided separating equilibrium the criterion does not impose belief restrictions on the out-of-equilibrium price constellations $\left(p_{I H}^{*}, p_{E L}^{*}\right)$ and $\left(p_{I L}^{*}, p_{E H}^{*}\right)$, under which 
the signals of the incumbent and the entrant appear contradictory. For these constellations it is not clear whether the incumbent or the entrant has deviated from his equilibrium strategy.

In what follows, we call a $\operatorname{PBE}\left(p^{*}, \mu^{*}(\cdot)\right)$ that satisfies the intuitive and the unprejudiced belief criterion a signalling equilibrium ${ }^{5}$ In the following section, we investigate the existence and properties of equilibria where prices reveal the entrant's quality.

\section{Informative Equilibria}

\section{One-Sided Separating Equilibria}

We start with the analysis of one-sided separating equilibria, in which one firm chooses a pooling and the other a separating pricing strategy. We will show that such equilibria typically do not exist, except for special parameter constellations. First, consider the case where the incumbent's price $p_{I}^{*}=p_{I L}^{*}=p_{I H}^{*}$ is independent of the entrant's quality, whereas the entrant chooses quality contingent prices $p_{E L}^{*}$ and $p_{E H}^{*}$ with $p_{E L}^{*} \neq p_{E H}^{*}$. Because in equilibrium the uninformed consumers infer the entrant's quality from his price, their beliefs satisfy $\mu^{*}\left(p_{I}^{*}, p_{E L}^{*}\right)=0$ and $\mu^{*}\left(p_{I}^{*}, p_{E H}^{*}\right)=1$. The following lemma establishes necessary conditions for this type of equilibrium.

Lemma 1 Suppose that the prices $p$, with $p_{I}=p_{I L}=p_{I H}, p_{E L} \neq p_{E H}$, can be supported as a signalling equilibrium $(p, \mu(\cdot))$ by some beliefs $\mu(\cdot)$. Then $p$ must satisfy

$$
\begin{gathered}
p_{E L}=\operatorname{argmax}_{p} \Pi_{E L}\left(p_{I}, p, 0\right), \\
p_{I}=\operatorname{argmax}_{p} \Pi_{I H}\left(p, p_{E H}, 1\right)=\operatorname{argmax}_{p} \Pi_{I L}\left(p, p_{E L}, 0\right), \\
p_{E H} \text { maximizes } \Pi_{E H}\left(p_{I}, p, 1\right) \text { subject to } \Pi_{E L}\left(p_{I}, p, 1\right) \leq \Pi_{E L}\left(p_{I}, p_{E L}, 0\right) .
\end{gathered}
$$

Condition (21) simply states that the low quality entrant's price reaction against $p_{I}$ is not distorted by signalling considerations. Indeed, some price $p$ not satisfying (21) can maximize the low quality seller's profit only if $\mu\left(p_{I}, p\right)>0$. But this is inconsistent with an equilibrium where prices reveal the true quality. The same argument underlies the first condition in (22) for the incumbent's price when competing against the high quality entrant. The incumbent's price reaction against $p_{E H}$ cannot be distorted because the consumers' belief that the entrant has high quality is already the worst possible belief from the incumbent's perspective.

The second condition for $p_{I}$ in (22) is implied by part (b) of the unprejudiced belief criterion. This criterion restricts the consumers' belief to $\mu\left(p, p_{E L}\right)=0$ for all $p \neq p_{I}$. Further, Bayes' rule in $(15)$ requires that $\mu\left(p_{I}, p_{E L}\right)=0$. Thus, the incumbent's pricing has no impact on consumer beliefs when facing the low quality entrant, and so in this situation there are also no signalling distortions.

\footnotetext{
${ }^{5}$ In our analysis the term 'unprejudiced belief criterion' always refers to the simplified version defined above.
} 
Finally, the constraint in condition (23) has to be satisfied because otherwise the low quality entrant would gain by imitating the high quality entrant's price. Further, the intuitive criterion implies that consumers infer high quality whenever the entrant gains by deviating to some price satisfying this constraint. Accordingly, the high quality entrant's price $p_{E H}$ must solve the constrained maximization problem in (23).

Lemma 1 allows us to show that a one-sided separating equilibrium with $p_{E L}^{*} \neq p_{E H}^{*}$ exists at most for a single value of the parameter $\gamma$. Since there is no reason for why the fraction of informed consumers should be identical to this value, an equilibrium of this type generically fails to exist.

Proposition 1 For all $\gamma \neq \max \{t /(t+\Delta), \Delta /(t+\Delta)\}$ there exists no signalling equilibrium $\left(p^{*}, \mu^{*}(\cdot)\right)$ such that $p_{I L}^{*}=p_{I H}^{*}$ and $p_{E L}^{*} \neq p_{E H}^{*}$.

The nonexistence result stated in Proposition 1 is a straightforward implication of Lemma 1. The lemma shows that prices in a one-sided separating equilibrium have to satisfy four conditions. Yet, such an equilibrium determines only three prices. This means that not all conditions can hold simultaneously, unless the exogenous parameters accidentally make one of the conditions redundant. The following lemma shows that a similar observation applies to the other type of one-sided separating equilibria, in which the entrant adopts a pooling strategy $p_{E}^{*}=p_{E L}^{*}=p_{E H}^{*}$ and only the incumbent's prices $p_{I L}^{*}$ and $p_{E H}^{*}$ reveal the entrant's quality so that $\mu^{*}\left(p_{E}^{*}, p_{I L}^{*}\right)=0$ and $\mu^{*}\left(p_{E}^{*}, p_{I H}^{*}\right)=1$.

Lemma 2 Suppose that the prices $p$, with $p_{I L} \neq p_{I H}, p_{E}=p_{E L}=p_{E H}$, can be supported as a signalling equilibrium $(p, \mu(\cdot))$ by some beliefs $\mu(\cdot)$. Then $p$ must satisfy

$$
\begin{gathered}
p_{I H}=\operatorname{argmax}_{p} \Pi_{I H}\left(p, p_{E}, 1\right), \\
p_{E}=\operatorname{argmax}_{p} \Pi_{E L}\left(p_{I L}, p, 0\right)=\operatorname{argmax}_{p} \Pi_{E H}\left(p_{I H}, p, 1\right), \\
p_{I L} \text { maximizes } \Pi_{I L}\left(p, p_{E}, 0\right) \text { subject to } \Pi_{I H}\left(p, p_{E}, 0\right) \leq \Pi_{I H}\left(p_{I H}, p_{E}, 1\right) .
\end{gathered}
$$

By our next proposition, the implications Lemmas 2 are similar to Lemma 1 . In fact, Lemma 2 implies that a one-sided separating equilibrium with $p_{I L}^{*} \neq p_{I H}^{*}$ typically fails to exist.

Proposition 2 For generic values of $\gamma$ there exists no signalling equilibrium $\left(p^{*}, \mu^{*}(\cdot)\right)$ such that $p_{I L}^{*} \neq p_{I H}^{*}$ and $p_{E L}^{*}=p_{E H}^{*}$.

Our results so far show that in an informative equilibrium it cannot happen that one of the duopolists adopts a pooling strategy. Propositions 1 and 2 eliminate one-sided pooling by combining the intuitive and the unprejudiced belief criterion. This leaves a two-sided separating equilibrium as the remaining candidate for a signalling equilibrium. 


\section{Two-Sided Separating Equilibria}

In a two-sided separating equilibrium the uninformed consumers' equilibrium belief is $\mu^{*}\left(p_{I L}^{*}, p_{E L}^{*}\right)=0$ and $\mu^{*}\left(p_{I H}^{*}, p_{E H}^{*}\right)=1$ as $p_{I L}^{*} \neq p_{I H}^{*}$ and $p_{E L}^{*} \neq p_{E H}^{*}$. Since each firm's price is informative, the intuitive criterion is no longer applicable. Therefore, only the unprejudiced belief criterion plays a role in the following lemma which provides necessary and sufficient conditions for a two-sided separating equilibrium.

Lemma 3 The prices $p$, with $p_{I L} \neq p_{I H}, p_{E L} \neq p_{E H}$, can be supported as a signalling equilibrium $(p, \mu(\cdot))$ by some beliefs $\mu(\cdot)$ if and only if

(a) $p$ is identical to the perfect information equilibrium $\hat{p}$, and

(b) there exists some $\bar{\mu} \in[0,1]$ such that

$$
\Pi_{I H}\left(p_{I H}, p_{E H}, 1\right) \geq \Pi_{I H}\left(p_{I L}, p_{E H}, \bar{\mu}\right), \quad \Pi_{E L}\left(p_{I L}, p_{E L}, 0\right) \geq \Pi_{E L}\left(p_{I L}, p_{E H}, \bar{\mu}\right) .
$$

By statement (a) of Lemma 3 , in a two-sided separating equilibrium the firms' prices are identical to the outcome of price competition under full information of all market participants about the entrant's quality. Thus, even though prices act as signals, they are not distorted by incentive restrictions. This observation is a well-known implication of the unprejudiced belief refinement (see Bagwell and Ramey (1991)) ${ }^{6}$ The idea is simply that the high quality entrant can ignore signalling effects when already the incumbent's price convinces the uninformed consumers of high quality. Similarly, the incumbent does not have to resort to distorted pricing to indicate a low quality entrant, because the entrant himself already reveals his quality through his price setting strategy. In a two-sided separating equilibrium, therefore, the firms' prices are determined as mutually undistorted best responses against the competitor and are thus identical to the full information equilibrium.

While prices are not distorted by signalling effects, statement (b) of Lemma 3 shows that they have to satisfy an incentive compatibility restriction, which is related to the signalling rivalry between the duopolists. The uninformed consumers will be perplexed when they observe the out-of-equilibrium price pair $\left(\hat{p}_{I L}, \hat{p}_{E H}\right)$. These prices are contradictory because the incumbent's price signals a low quality entrant and the entrant's price a high quality. Also, it is not clear which firm has deviated from its equilibrium strategy. The prices $\left(\hat{p}_{I L}, \hat{p}_{E H}\right)$ could originate from the equilibrium pair $\left(\hat{p}_{I H}, \hat{p}_{E H}\right)$ because the incumbent has deviated to $\hat{p}_{I L}$; or they could originate from the equilibrium pair $\left(\hat{p}_{I L}, \hat{p}_{E L}\right)$ because the entrant has deviated to $\hat{p}_{E H}$.

Condition (27) states that there must be some belief $\bar{\mu}=\mu\left(\hat{p}_{I L}, \hat{p}_{E H}\right)$ that deters both kinds of deviations. On the one hand, by the first inequality in $(27), \bar{\mu}$ must be high enough so as to make it unattractive for the incumbent to deviate from $\hat{p}_{I H}$ to $\hat{p}_{I L}$. On the other

\footnotetext{
${ }^{6}$ Yehezkel (2006) proposes a generalization of the unprejudiced belief criterion that eliminates all possible separating equilibria but the full information outcome.
} 


\begin{tabular}{|l||c|c|c|c|c|c|}
\hline$\left.\bar{\gamma}\right|_{\Delta=10}$ & $t=2$ & $t=4$ & $t=6$ & $t=8$ & $t=10$ & $t=12$ \\
\hline \hline$c=5$ & 0.870 & 0.700 & 0.645 & 0.662 & 0.705 & 0.738 \\
\hline$c=7$ & 0.775 & 0.736 & 0.657 & 0.587 & 0.634 & 0.671 \\
\hline$c=9$ & 0.846 & 0.736 & 0.645 & 0.572 & 0.548 & 0.590 \\
\hline
\end{tabular}

Table 1: Numerical values for $\bar{\gamma}$.

hand, the second inequality in (27) requires that $\bar{\mu}$ is small enough so that the entrant cannot gain by deviating from $\hat{p}_{E L}$ to $\hat{p}_{E H}$. To examine whether both inequalities can be satisfied, we first consider the case of non-drastic innovations $(\Delta-c<3 t)$ and then drastic innovations $(\Delta-c \geq 3 t)$.

\section{Non-drastic Innovations}

Whether condition (b) of Lemma 3 is satisfied for $p=\hat{p}$, depends on how large the fraction $\gamma$ of informed consumers is. As the proof of our next result shows, Lemma 3 implies that a two-sided separating equilibrium exists if and only if $\gamma$ is not too small.

Proposition 3 Let $\Delta-c<3 t$. There exists a $\bar{\gamma}=\bar{\gamma}(t, \Delta, c) \in(0,1)$ such that the following holds:

(a) If $\gamma \geq \bar{\gamma}$, then there exists a signalling equilibrium $\left(p^{*}, \mu^{*}(\cdot)\right)$ with $p_{I L}^{*} \neq p_{I H}^{*}$ and $p_{E L}^{*} \neq p_{E H}^{*}$. The prices $p^{*}$ in this equilibrium are identical to the perfect information equilibrium $\hat{p}$.

(b) If $\gamma<\bar{\gamma}$, there exists no signalling equilibrium $\left(p^{*}, \mu^{*}(\cdot)\right)$ such that $p_{I L}^{*} \neq p_{I H}^{*}$ and $p_{E L}^{*} \neq p_{E H}^{*}$.

In a two-sided separating equilibrium prices are not distorted by signalling. The incumbent or the entrant can gain by a unilateral deviation only because this changes the uninformed consumers' beliefs. Therefore, a deviation is unprofitable as long as sufficiently many consumers are informed. This explains why $\left(\hat{p}, \mu^{*}(\cdot)\right)$ can constitute a signalling equilibrium for $\gamma \geq \bar{\gamma}$. If $\gamma<\bar{\gamma}$, then the firms' signalling rivalry is too intense to prevent profitable deviations: Either the incumbent will defect from the equilibrium if $q_{E}=q_{H}$, or the entrant will defect if $q_{E}=q_{L}$. As observed by Schultz (1999) in a different context, conflicting interests may thus rule out the existence of a two-sided separating equilibrium for some parameter constellations.

In Table 1 some numerical calculations illustrate how $\bar{\gamma}$ depends on $c$ and $t$ if $\Delta=10$. They reveal that $\bar{\gamma}$ does not depend monotonically on the parameters of our model. In particular, if the measure $t$ of horizontal differentiation increases, $\bar{\gamma}$ first decreases and then increases. Our next result shows that this is true in general. 
Proposition 4 (a) For $t$ sufficiently large the critical value $\bar{\gamma}(t, \Delta, c)$, stated in Proposition 3 . satisfies

$$
\frac{\partial \bar{\gamma}(t, \Delta, c)}{\partial t}>0,\left.\quad \frac{\partial \bar{\gamma}(t, \lambda \Delta, \lambda c)}{\partial \lambda}\right|_{\lambda=1}<0
$$

(b) For $t$ sufficiently close to $(\Delta-c) / 3,7$

$$
\frac{\partial \bar{\gamma}(t, \Delta, c)}{\partial t}<0,\left.\quad \frac{\partial \bar{\gamma}(t, \lambda \Delta, \lambda c)}{\partial \lambda}\right|_{\lambda=1}>0
$$

Prices can be used as credible signals because of their effect on the informed consumers' demand. When the degree $t$ of horizontal differentiation is sufficiently large, some of these consumers still purchase from the firm that has deviated to a higher price. In this situation, $\bar{\gamma}$ is increasing in $t$ because the price sensitivity of demand is negatively related to the product differentiation parameter $t$. Conversely, fewer informed consumers are required for the twosided separating equilibrium if $\Delta-c$ is increased to $\lambda \Delta-\lambda c$, with $\lambda>1$. Such an increase improves the competitiveness of the high quality entrant. It facilitates the requirements for an equilibrium because it raises the price differences $\left|\hat{p}_{I H}-\hat{p}_{I L}\right|$ and $\left|\hat{p}_{E L}-\hat{p}_{E H}\right|$. Therefore, a deviation of the incumbent from $\hat{p}_{I H}$ to $\hat{p}_{I L}$ or of the entrant from $\hat{p}_{E L}$ to $\hat{p}_{E H}$ becomes less profitable, and a smaller fraction $\bar{\gamma}$ of informed consumers suffices for existence of a signalling equilibrium.

As part (b) of Proposition 4 shows, the comparative statics properties of $\bar{\gamma}$ are reversed if $t$ is relatively small. Then, by deviating from the full information price to a higher price, a firm loses the entire demand of the informed consumers. Therefore, the price sensitivity of the informed consumers' demand plays no role. Instead, because equilibrium profits are increasing in $t$, the incentives for deviation become weaker if $t$ increases. In particular, the incumbent is driven out of the market by the high quality entrant and receives zero profits in the limit as $t \rightarrow(\Delta-c) / 3$. Therefore, when $t$ becomes small more informed consumers are required to prevent the incumbent from deviating to $\hat{p}_{I L}$.

\section{Drastic Innovations}

Now we show that Proposition 3 holds also for drastic innovations: if the fraction $\gamma$ of informed consumers is not too small, then the full information prices $\hat{p}$ in (12) can be supported as a signalling equilibrium by some beliefs $\mu^{*}(\cdot)$. We begin with the following lemma:

Lemma 4 Let $\Delta-c>3 t$. If and only if $\bar{\mu} \geq(\Delta-t) / \Delta$, then

$$
\Pi_{I H}\left(\hat{p}_{I H}, \hat{p}_{E H}, 1\right) \geq \Pi_{I H}\left(\hat{p}_{I L}, \hat{p}_{E H}, \bar{\mu}\right)
$$

i.e. the incumbent does not gain from deviating to $\hat{p}_{I L}$ if $q_{E}=q_{H}$.

\footnotetext{
${ }^{7}$ Recall that $t>(\Delta-c) / 3$
} 
Since the incumbent makes zero profit at the prices $\left(\hat{p}_{I H}, \hat{p}_{E H}\right)$, he could gain by deviating to $\hat{p}_{I L}$ only if this attracts some uninformed consumers. Lemma 4 shows that this is not possible as long as the uninformed consumers remain sufficiently optimistic about the entrant's quality when observing the out-of-equilibrium prices $\left(\hat{p}_{I L}, \hat{p}_{E H}\right)$.

Lemma 5 Let $\Delta-c>3 t$ and $\bar{\mu} \geq(\Delta-t) / \Delta$. If and only if $\gamma \geq(2 \Delta-3 t) /(2 \Delta-2 t)$, then

$$
\Pi_{E L}\left(\hat{p}_{I L}, \hat{p}_{E L}, 0\right) \geq \Pi_{E L}\left(\hat{p}_{I L}, \hat{p}_{E H}, \bar{\mu}\right) .
$$

i.e. the entrant does not gain from deviating to $\hat{p}_{E H}$ if $q_{E}=q_{L}$.

By deviating to $\hat{p}_{E H}$ the low quality entrant loses the demand from the informed consumers. But, by Lemma 4 he attracts all uninformed consumers. A deviation is therefore not profitable only if the fraction of informed consumers is not too small.

Lemmas 3, 4 and 5 immediately imply the following extension of Proposition 3 to the case of drastic innovations:

Proposition 5 Let $\Delta-c>3 t$ and define $\tilde{\gamma} \equiv(2 \Delta-3 t) /(2 \Delta-2 t)$.

(a) If $\gamma \geq \tilde{\gamma}$, there exists a signalling equilibrium $\left(p^{*}, \mu^{*}(\cdot)\right)$ with $p_{I L}^{*} \neq p_{I H}^{*}$ and $p_{E L}^{*} \neq p_{E H}^{*}$. The prices $p^{*}$ in this equilibrium are identical to the perfect information equilibrium $\hat{p}$.

(b) If $\gamma<\tilde{\gamma}$, there exists no signalling equilibrium $\left(p^{*}, \mu^{*}(\cdot)\right)$ such that $p_{I L}^{*} \neq p_{I H}^{*}$ and $p_{E L}^{*} \neq p_{E H}^{*}$.

Since $\partial \tilde{\gamma} / \partial t<0$ and $\partial \tilde{\gamma} / \partial \Delta>0$, the comparative statics properties of $\tilde{\gamma}$ are in line with part (b) of Proposition 4. Interestingly, in the limit $t \rightarrow 0$ we obtain $\tilde{\gamma} \rightarrow 1$. This means that in a homogenous market a separating signalling equilibrium cannot exist with some uninformed consumers. The intuition is that the incumbent is driven out of the market by the high quality entrant, and for $t \rightarrow 0$ also the low quality entrant's profits become negligible at the full information prices. Therefore, if $q_{E}=q_{H}$ the incumbent will deviate from $p_{I H}^{*}$ to $p_{I L}^{*}$ if this attracts some uninformed consumers. Should no uninformed consumer purchase from the incumbent, this means that all uninformed consumers turn to the entrant when observing the prices $\left(p_{I L}^{*}, p_{E H}^{*}\right)$. But this implies that the low quality entrant will gain by deviating from $p_{E L}^{*}$ to $p_{E H}^{*}$.

\section{Conclusion}

Our analysis shows that a firm may not have to resort to distorted pricing to signal its quality to the uninformed consumers. If its quality is known to a competitor, then the prices of both firms become quality signals and signalling competition may lead to non-distorted pricing in equilibrium. Indeed, under two belief refinements that have frequently been used in the 
literature, we show that prices convey quality information only if the are identical to the equilibrium under full information.

This finding has obvious implications for other strategic choices. For example, consider the market entry decision of a firm whose quality is not publicly observable. As long as sufficiently many consumers are informed, our analysis indicates that entry decisions are not distorted when at least one of the incumbent firms learns the new firm's quality after it has entered the market. A similar conclusion obtains for $R \& D$ investments in product innovation when some consumers cannot observe whether the investment has been successful or not. If competing firms and a sufficient fraction of consumers become informed about the outcome, our results suggest that the incentives for product innovation are not distorted by the presence of uninformed consumers. 


\section{Appendix}

Proof of Lemma 1: Since $p_{E L} \neq p_{E H}$ implies $\mu\left(p_{I}, p_{E L}\right)=0$ and $\partial \Pi_{E L} / \partial \mu>0$, it follows from equilibrium condition (14) that for all $p \geq 0$

$$
\Pi_{E L}\left(p_{I}, p_{E L}, 0\right) \geq \Pi_{E L}\left(p_{I}, p, \mu\left(p_{I}, p\right)\right) \geq \Pi_{E L}\left(p_{I}, p, 0\right) .
$$

This proves that (21) must hold. Analogously, $\mu\left(p_{I}, p_{E H}\right)=1$ and $\partial \Pi_{I H} / \partial \mu<0$ imply by (13) that for all $p \geq 0$

$$
\Pi_{I H}\left(p_{I}, p_{E H}, 1\right) \geq \Pi_{I H}\left(p, p_{E H}, \mu\left(p, p_{E H}\right)\right) \geq \Pi_{I H}\left(p, p_{E H}, 1\right) .
$$

This proves that $p_{I}$ must satisfy the first condition in (22).

Suppose that $p_{I}$ does not satisfy the second condition in (22). Since part (b) of the unprejudiced belief criterion implies $\mu\left(p, p_{E L}\right)=0$ for all $p \neq p_{I}$, then there exist some $p$ such that

$$
\Pi_{I L}\left(p_{I}, p_{E L}, \mu\left(p_{I}, p_{E L}\right)\right)=\Pi_{I L}\left(p_{I}, p_{E L}, 0\right)<\Pi_{I L}\left(p, p_{E L}, 0\right)=\Pi_{I L}\left(p, p_{E L}, \mu\left(p, p_{E L}\right)\right) .
$$

This is a contradiction to the condition that in equilibrium $p_{I}$ has to satisfy $(13)$ for $Q=L$.

Note that $p_{E H}$ must satisfy the constraint in (23) because equilibrium condition (14) implies that

$$
\Pi_{E L}\left(p_{I}, p_{E L}, 0\right)=\Pi_{E L}\left(p_{I}, p_{E L}, \mu\left(p_{I}, p_{E L}\right)\right) \geq \Pi_{E L}\left(p_{I}, p_{E H}, \mu\left(p_{I}, p_{E H}\right)\right)=\Pi_{E L}\left(p_{I}, p_{E H}, 1\right) .
$$

Suppose that $p_{E H}$ does not solve the maximization problem in (23). Then there exists some $p$ that satisfies the constraint in (23) and $\Pi_{E H}\left(p_{I}, p, 1\right)>\Pi_{E H}\left(p_{I}, p_{E H}, 1\right)$. Because part (b) of the intuitive criterion then implies $\mu\left(p_{I}, p\right)=1$, this yields

$$
\Pi_{E H}\left(p_{I}, p, \mu\left(p_{I}, p\right)\right)=\Pi_{E H}\left(p_{I}, p, 1\right)>\Pi_{E H}\left(p_{I}, p_{E H}, 1\right)=\Pi_{E H}\left(p_{I}, p_{E H}, \mu\left(p_{I}, p_{E H}\right)\right),
$$

a contradiction to equilibrium condition (14) for $Q=H$.

Q.E.D.

Proof of Proposition 1: Using the full-information best-response functions to solve the conditions for (21) and (22) in Lemma 1, we get the prices

$$
p_{I}^{*}=t, p_{E L}^{*}=t, p_{E H}^{*}=t+\Delta .
$$

If the constraint in (23) is not binding, we obtain the high-quality entrant's best-response

$$
p_{E H}^{*}=\max \left\{\frac{2 t+\Delta+c}{2}, \Delta\right\} .
$$

This, however, is inconsistent with the last equation in (37) as $\Delta>c$ and $t>0$. If the constraint in (23) is binding, then $\Pi_{E L}\left(p_{I}^{*}, p_{E H}^{*}, 1\right)=\Pi_{E L}\left(p_{I}^{*}, p_{E L}^{*}, 0\right)$. By (37) this equality is equivalent to

$$
\frac{(\Delta+t)(t-\gamma \Delta)}{2 t}=\frac{t}{2}
$$


for $\Delta \leq t$ and

$$
\frac{(\Delta+t)(1-\gamma)}{2}=\frac{t}{2}
$$

for $\Delta>t$. From these equations it follows that the conditions of Lemma 1 are satisfied only if $\gamma=t /(t+\Delta)$ if $\Delta \leq t$ and $\gamma=\Delta /(t+\Delta)$ if $\Delta>t$.

Proof of Lemma 2: The argument is analogous to the proof of Lemma 1 .

Proof of Proposition 2: From the full-information best responses for (24) and (25) in Lemma 2, we obtain the solution

$$
p_{I L}^{*}=\frac{3 t+2 \Delta+4 c}{3}, p_{I H}^{*}=\frac{3 t-\Delta+c}{3}, p_{E}^{*}=\frac{3 t+\Delta+2 c}{3}
$$

for $\Delta-c \leq 3 t$ and

$$
p_{I L}^{*}=2 \Delta-3 t, p_{I H}^{*}=0, p_{E}^{*}=\Delta-t
$$

for $\Delta-c>3 t$.

If the constraint in (26) is not binding and $\Delta-c \leq 3 t$, the full-information best-response of $I L$ is given by $p_{I L}^{*}=\max \{(6 t+\Delta+2 c) / 6,(\Delta+2 c) / 3\}$. This, however, is inconsistent with the first equation in (41). If the constraint in (26) is not binding and $\Delta-c>3 t$, the full-information best-response of $I L$ is given by $p_{I L}^{*}=\max \{\Delta / 2, \Delta-2 t\}$. This, however, is inconsistent with the first equation in (42).

For $\Delta-c<3 t$, if the constraint in (26) is not binding, the full-information best-response of $I L$ is given by $p_{I L}^{*}=\max \{(6 t+\Delta+2 c) / 6,(\Delta+2 c) / 3\}$. This, however, is inconsistent with the first equation in (41). If the constraint in $(26)$ is binding, then $\Pi_{I H}\left(p_{I L}^{*}, p_{E}^{*}, 0\right)=$ $\Pi_{I H}\left(p_{I H}^{*}, p_{E}^{*}, 1\right)$. If $\Delta-c<3 t \leq \Delta+2 c$, this equality is by (41) equivalent to

$$
0=\frac{(3 t-\Delta+c)^{2}}{18 t} \text {. }
$$

If $\Delta+2 c<3 t \leq 4 \Delta+2 c$, then the equality $\Pi_{I H}\left(p_{I L}^{*}, p_{E}^{*}, 0\right)=\Pi_{I H}\left(p_{I H}^{*}, p_{E}^{*}, 1\right)$ by (41) is equivalent to

$$
\frac{(1-\gamma)(3 t+2 \Delta+4 c)(3 t-\Delta-2 c)}{18 t}=\frac{(3 t-\Delta+c)^{2}}{18 t} \text {. }
$$

Finally, if $4 \Delta+2 c \leq 3 t$, then by (41) then the equality $\Pi_{I H}\left(p_{I L}^{*}, p_{E}^{*}, 0\right)=\Pi_{I H}\left(p_{I H}^{*}, p_{E}^{*}, 1\right)$ is equivalent to

$$
\frac{(3 t+2 \Delta+4 c)(3 t-3 \gamma \Delta-\Delta-2 c)}{18 t}=\frac{(3 t-\Delta+c)^{2}}{18 t} .
$$

As $\Delta-c<3 t$, the equations (43) - 45) have either no solution or a unique solution in $\gamma$ Therefore, the conditions of Lemma 2 can hold at most for particular values of $\gamma$. Q.E.D. Proof of Lemma 3: We first show that (a) and (b) must hold in a signalling equilibrium $(p, \mu(\cdot))$. By $(13)$

$$
\Pi_{I H}\left(p_{I H}, p_{E H}, 1\right) \geq \Pi_{I H}\left(p, p_{E H}, \mu\left(p, p_{E H}\right)\right) \geq \Pi_{I H}\left(p, p_{E H}, 1\right)
$$


for all $p \geq 0$, where the second inequality follows from $\partial \Pi_{I H} / \partial \mu<0$. Similarly, (13) and part (b) of the unprejudiced belief criterion imply

$$
\Pi_{I L}\left(p_{I L}, p_{E L}, 0\right) \geq \Pi_{I L}\left(p, p_{E L}, \mu\left(p, p_{E L}\right)\right)=\Pi_{I L}\left(p, p_{E L}, 0\right)
$$

for all $p \neq p_{I H}$. By continuity of $\Pi_{I L}\left(\cdot, p_{E L}, 0\right)$, therefore also

$$
\Pi_{I L}\left(p_{I L}, p_{E L}, 0\right) \geq \Pi_{I L}\left(p_{I H}, p_{E L}, 0\right) .
$$

By an analogous argument it follows from (13), $\partial \Pi_{E L} / \partial \mu>0$, and part (a) of the unprejudiced belief criterion that

$$
\Pi_{E L}\left(p_{I L}, p_{E L}, 0\right) \geq \Pi_{E L}\left(p_{I L}, p, 0\right), \quad \Pi_{E H}\left(p_{I H}, p_{E H}, 1\right) \geq \Pi_{E H}\left(p_{I H}, p, 1\right)
$$

for all $p \geq 0$. By (46)-(49), $p$ satisfies the conditions that define $\hat{p}$ in (7). This proves that $(p, \mu(\cdot))$ must satisfy claim (a) that $p=\hat{p}$. Note that by (13) and (14)

$$
\begin{aligned}
\Pi_{I H}\left(p_{I H}, p_{E H}, 1\right) & \geq \Pi_{I H}\left(p_{I L}, p_{E H}, \mu\left(p_{I L}, p_{E H}\right)\right), \\
\Pi_{E L}\left(p_{I L}, p_{E L}, 0\right) & \geq \Pi_{E L}\left(p_{I L}, p_{E H}, \mu\left(p_{I L}, p_{E H}\right)\right) .
\end{aligned}
$$

This proves that statement (b) holds for $\bar{\mu} \equiv \mu\left(p_{I L}, p_{E H}\right)$.

Next we show that $(\hat{p}, \mu(\cdot))$ is a signalling equilibrium for some $\mu(\cdot)$ only if (b) holds. Note that the intuitive criterion does not apply to $\hat{p}$ because $\hat{p}_{I L} \neq \hat{p}_{I H}$ and $\hat{p}_{E L} \neq \hat{p}_{E H}$. In line with the unprejudiced belief criterion, define

$$
\mu\left(\hat{p}_{I H}, p\right) \equiv 1 \text { for all } p \neq \hat{p}_{E L}, \quad \mu\left(p, \hat{p}_{E L}\right) \equiv 0 \text { for all } p \neq \hat{p}_{I H}, \quad \mu\left(\hat{p}_{I H}, \hat{p}_{E L}\right) \equiv \lambda .
$$

Further, if (27) in part (b) of the lemma holds for $p=\hat{p}$ we can set

$$
\mu\left(\hat{p}_{I L}, p\right) \equiv 0 \text { for all } p \neq \hat{p}_{E H}, \quad \mu\left(p, \hat{p}_{E H}\right) \equiv 1 \text { for all } p \neq \hat{p}_{I L}, \quad \mu\left(\hat{p}_{I L}, \hat{p}_{E H}\right) \equiv \bar{\mu} .
$$

The beliefs for all other price pairs $\left(p_{I}, p_{E}\right)$ play no role in the definition of a PBE and so they are arbitrary. Since $\mu\left(\hat{p}_{I L}, \hat{p}_{E L}\right)=0$ and $\mu\left(\hat{p}_{I H}, \hat{p}_{E H}\right)=1$ by (51) and (52), these beliefs satisfy Bayes rule (15) in part (b) of the definition of a PBE. Further since $\hat{p}$ satisfies (7) and (50) holds for $p=\hat{p}$, it is easily verified that $(\hat{p}, \mu(\cdot))$ satisfies also the conditions (13) and (14) for profit maximization in part (a) of the definition of a PBE. This proves that $\hat{p}$ and the beliefs $\mu(\cdot)$ in (51) and (52) constitute a signalling equilibrium if (27) in part (b) of the lemma holds for $p=\hat{p}$. If the latter condition does not hold, then there is no belief $\mu\left(p_{I L}, p_{E H}\right)$ that satisfies both conditions in (50) for $p=\hat{p}$. In this case, there exists no $\operatorname{PBE}(p, \mu(\cdot))$ with $p=\hat{p}$ because at least one of the conditions (13) and (14) for profit maximization is violated.

Q.E.D.

Proof of Proposition 3: By Lemma 3 it is sufficient to show that for $p=\hat{p}$ (27) has a solution $\bar{\mu} \in[0,1]$ if and only if $\gamma \geq \bar{\gamma}$. Using $\hat{p}$ in $(12)$, we have $\Pi_{I H}\left(\hat{p}_{I H}, \hat{p}_{E H}, 1\right)=(3 t-\Delta+c)^{2} /(18 t)$ and

$$
\Pi_{I H}\left(\hat{p}_{I L}, \hat{p}_{E H}, \bar{\mu}\right)=\frac{(1-\gamma)(\Delta(1-3 \bar{\mu})+2 c+3 t)}{6}+\min \left[0, \frac{\gamma(3 t-2 \Delta-2 c)}{6}\right],
$$


because if $q_{E}=q_{H}$ the incumbent's demand from the informed consumers is zero at $\left(\hat{p}_{I L}, \hat{p}_{E H}\right)$ for $3 t \leq 2(\Delta-c)$. Therefore, solving the the first inequality in $(27)$ for $\bar{\mu}$ yields

$$
\bar{\mu} \geq \bar{\mu}_{I}(\gamma) \equiv \frac{9 t \Delta(1-\gamma)-(\Delta-c)^{2}}{9 t \Delta(1-\gamma)}+\max \left[0, \frac{\gamma}{1-\gamma} \frac{2 \Delta-2 c-3 t}{3 \Delta}\right] .
$$

By (12), we have $\Pi_{E L}\left(\hat{p}_{I L}, \hat{p}_{E L}, 0\right)=t / 2$ and

$$
\begin{aligned}
\Pi_{E L}\left(\hat{p}_{I L}, \hat{p}_{E H}, \bar{\mu}\right)= & \frac{(1-\gamma)(3 t+2 c+\Delta)(3 t-2 c+\Delta(3 \bar{\mu}-1))}{18 t} \\
& +\min \left[0, \frac{\gamma\left[(3 t)^{2}-(\Delta+2 c)^{2}\right]}{18 t}\right],
\end{aligned}
$$

because if $q_{E}=q_{L}$ the entrant's demand from the informed consumers is zero at $\left(\hat{p}_{I L}, \hat{p}_{E H}\right)$ for $3 t \leq \Delta+2 c$. Therefore, solving the the second inequality in (27) for $\bar{\mu}$ yields

$$
\bar{\mu} \leq \bar{\mu}_{E}(\gamma) \equiv \frac{(\Delta+2 c)^{2}}{3 \Delta(1-\gamma)(3 t+2 c+\Delta)}-\max \left[0, \frac{\gamma}{1-\gamma} \frac{\Delta+2 c-3 t}{3 \Delta}\right] .
$$

Thus, the two inequalities in (27) admit a solution $\bar{\mu}$ if and only if $\bar{\mu}_{I}(\gamma) \leq \bar{\mu}_{E}(\gamma)$. It is easily verified that

$$
0<\bar{\mu}_{E}(0)<\bar{\mu}_{I}(0)<1, \lim _{\gamma \rightarrow 1}\left[\bar{\mu}_{I}(\gamma)-\bar{\mu}_{E}(\gamma)\right]<0, \bar{\mu}_{I}^{\prime}(\gamma)<0, \bar{\mu}_{E}^{\prime}(\gamma)>0 .
$$

Therefore, by the intermediate value theorem there exist a (unique) $\bar{\gamma} \in(0,1)$ such that $\bar{\mu}_{I}(\bar{\gamma})=\bar{\mu}_{E}(\bar{\gamma}) \in(0,1)$ and $\bar{\mu}_{I}(\gamma) \leq \bar{\mu}_{E}(\gamma)$ if and only if $\gamma \geq \bar{\gamma}$.

Q.E.D.

Proof of Proposition 4: By the proof of Proposition 3, $\bar{\gamma}$ is uniquely determined by the solution of $\bar{\mu}_{I}(\bar{\gamma} \mid t, \Delta, c)=\bar{\mu}_{E}(\bar{\gamma} \mid t, \Delta, c)$, where $\bar{\mu}_{I}$ and $\bar{\mu}_{E}$ are defined in (54) and (56) and satisfy (57). For $3 t>\max [2(\Delta-c), \Delta+2 c]$ one obtains

$$
\partial \bar{\mu}_{I}(\bar{\gamma} \mid t, \Delta, c) / \partial t>0, \quad \partial \bar{\mu}_{E}(\bar{\gamma} \mid t, \Delta, c) / \partial t<0,
$$

which immediately implies the first inequality in (28). Since $\bar{\mu}_{I}(\bar{\gamma} \mid \lambda t, \lambda \Delta, \lambda c)=\bar{\mu}_{I}(\bar{\gamma} \mid t, \Delta, c)$ and $\bar{\mu}_{E}(\bar{\gamma} \mid \lambda t, \lambda \Delta, \lambda c)=\bar{\mu}_{E}(\bar{\gamma} \mid t, \Delta, c)$, the first inequality in (28) implies the second inequality in (28). This proves part (a).

To prove part (b), let $\Delta-c<3 t<\min [2(\Delta-c), \Delta+2 c]$. Then solving $\bar{\mu}_{I}(\bar{\gamma} \mid t, \Delta, c)=$ $\bar{\mu}_{E}(\bar{\gamma} \mid t, \Delta, c)$ yields

$$
\bar{\gamma}=\frac{\Delta\left(27 t^{2}+3 t \Delta-\Delta^{2}\right)+12 c t \Delta+3 c^{2}(\Delta-5 t)-2 c^{3}}{18 t^{2}(3 t+2 c+\Delta)} .
$$

Since

$$
\lim _{t \rightarrow(\Delta-c) / 3} \frac{\partial \bar{\gamma}}{\partial t}=-\frac{3(\Delta+2 c)}{2(2 \Delta+c)^{2}}<0
$$


this proves the first inequality in (29). As $\bar{\gamma}$ in (59) is homogenous of degree zero in $(t, \Delta, c)$ the first inequality implies the second inequality in (29).

Q.E.D.

Proof of Lemma 4: Note that $\Pi_{I H}\left(\hat{p}_{I H}, \hat{p}_{E H}, 1\right)=0$ because $\hat{p}_{I H}=0$. Therefore (30) holds if and only if no consumer purchases from the incumbent upon observing $\left(\hat{p}_{I L}, \hat{p}_{E H}\right)$. The uninformed consumers' demand is zero if and only if even the consumer with $x=0$ does not prefer to buy from the incumbent. This is the case if

$$
q_{L}-\hat{p}_{I L} \leq \bar{\mu} q_{H}+(1-\bar{\mu}) q_{L}-\hat{p}_{E H}-t
$$

Since $\hat{p}_{E H}-\hat{p}_{I L}=\Delta-2 t$, this is identical to $\bar{\mu} \geq(\Delta-t) / \Delta$. Clearly, if even the uninformed consumers do not purchase from the incumbent, then at the prices $\left(\hat{p}_{I L}, \hat{p}_{E H}\right)$ also the informed consumer do not buy from the incumbent because they know that $q_{E}=q_{H}$.

Q.E.D.

Proof of Lemma 5: We first show that no informed consumer purchases from the low quality entrant if he deviates to $p_{E H}^{*}$. This is the case if even the informed consumer with $x=1$ does not buy from the entrant, i.e. if

$$
q_{L}-\hat{p}_{I L}-t \geq q_{L}-\hat{p}_{E H} .
$$

Since $\hat{p}_{E H}-\hat{p}_{I L}=\Delta-2 t$, this is identical to $\Delta \geq 3 t$ and is satisfied as $\Delta-c \geq 3 t$.

While a deviation to $p_{E H}^{*}$ leads to zero demand from the informed consumers, by the proof of Lemma 4 all uninformed consumers buy from the entrant at the prices $\left(\hat{p}_{I L}, \hat{p}_{E H}\right)$. Therefore,

$$
\Pi_{E L}\left(\hat{p}_{I L}, \hat{p}_{E H}, \bar{\mu}\right)=(1-\gamma) \hat{p}_{E H}=(1-\gamma)(\Delta-t) .
$$

Since $\Pi_{E L}\left(\hat{p}_{I L}, \hat{p}_{E L}, 0\right)=\Pi_{E L}(t, t, 0)=t / 2$, the inequality in (31) is equivalent to $\gamma \geq(2 \Delta-$ $3 t) /(2 \Delta-2 t)$.

Q.E.D.

Proof of Proposition 4: Statements (a) and (b) follow immediately from Lemmas 3, 4 and 5.

Q.E.D. 


\section{References}

BAgwell, K. AND G. Ramey, "Oligopoly Limit Pricing." Rand Journal of Economics 22, (1991), 155-172.

Bagwell, K. And M. H. Riordan, "High and Declining Prices Signal Product Quality." American Economic Review 81, (1991), 224-239.

Bester, H., "Bargaining vs. Price Competition in Markets with Quality Uncertainty." American Economic Review 83, (1993), 278-288.

Bester, H. And K. Ritzberger, "Strategic Pricing, Signalling, and Costly Information Acquisition." International Journal of Industrial Organization 19, (2001), 1347-1361.

Cho, I.-K., And D. M. KRePs, "Signaling Games and Stable Equilibria." Quarterly Journal of Economics 102, (1987), 179-221.

Daughety A. F. And J. F. Reinganum, "Competition and Confidentiality: Signaling Quality in a Duopoly when there is Universal Private Information." Games and Economic Behavior 58, (2007), 94-120.

Daughety A. F. and J. F. Reinganum, "Imperfect Competition and Quality Signalling." RAND Journal of Economics 39, (2008), 163-183.

Fluet, C. AND P. G. Garella, "Advertising and Prices as Signals of Quality in a Regime of Price Rivalry." International Journal of Industrial Organization 20, (2002), 907-930.

Hertzendorf, M. N. and P. B. Overgaard, "Price Competition and Advertising Signals: Signaling by Competing Senders." Journal of Economics \& Management Strategy 10, (2001a), 621-662.

Hertzendorf, M. N. And P. B. Overgaard, "Prices as Signals of Quality in Duopoly." Working Paper, School of Economics and Management, University of Aarhus, (2001b).

Hotelling, H., "Stability in Competition." Economic Journal 39, (1929), 41-57.

Janssen, M. C. W. And S. Roy, "Signaling Quality through Prices in an Oligopoly." Games and Economic Behavior 68, (2010), 192-207.

Linnemer L., "Price and Advertising as Signals of Quality when some Consumers are Informed." International Journal of Industrial Organization 20, (2002), 931-947.

Milgrom P. And J. Roberts, "Price and Advertising Signals of Product Quality." Journal of Political Economy 94, (1986), 796-821.

Nelson, P., "Information and Consumer Behavior." Journal of Political Economy 78, (1970), 311-329. 
Schultz, C., "Limit Pricing when Incumbents have Conflicting Interests." International Journal of Industrial Organization 17, (1999), 801-825.

Shaked, A. And J. Sutton, "Relaxing Price Competition Through Product Differentiation." Review of Economic Studies 49, (1982), 3-13.

Yehezkel, Y., "On the Robustness of the Full-Information Separating Equilibrium in MultiSender Signaling Games." Tel Aviv University (mimeo), (2006).

Yehezkel, Y., "Signaling Quality in an Oligopoly when Some Consumers are Informed." Journal of Economics and Management Strategy 17, (2008), 937-972. 